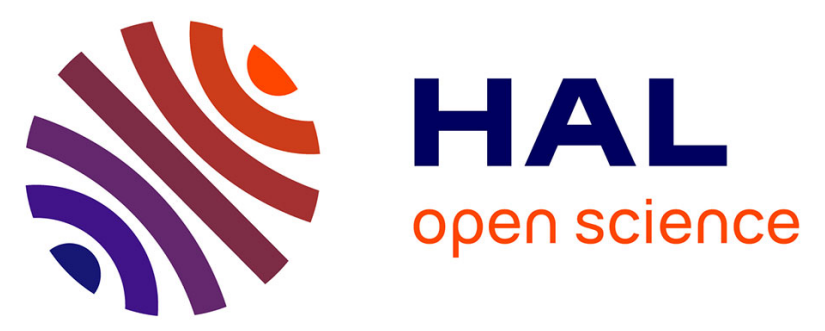

\title{
Unexpected giant elasticity in side-chain liquid-crystal polymer melts: A new approach for the understanding of shear-induced phase transitions
}

H Mendil, P Baroni, Laurence Noirez

\section{- To cite this version:}

H Mendil, P Baroni, Laurence Noirez. Unexpected giant elasticity in side-chain liquid-crystal polymer melts: A new approach for the understanding of shear-induced phase transitions. EPL - Europhysics Letters, 2005, 72 (6), pp.983-989. 10.1209/epl/i2004-10552-3 . hal-01363182

\section{HAL Id: hal-01363182 \\ https://hal.science/hal-01363182}

Submitted on 9 Sep 2016

HAL is a multi-disciplinary open access archive for the deposit and dissemination of scientific research documents, whether they are published or not. The documents may come from teaching and research institutions in France or abroad, or from public or private research centers.
L'archive ouverte pluridisciplinaire HAL, est destinée au dépôt et à la diffusion de documents scientifiques de niveau recherche, publiés ou non, émanant des établissements d'enseignement et de recherche français ou étrangers, des laboratoires publics ou privés. 


\title{
Unexpected Giant Elasticity in Side-Chain Liquid-Crystal Polymer melts: A new approach for understanding of shear induced phase transitions.
}

\author{
H. MENDIL, P. BARONI, L. NOIREZ \\ Laboratoire Léon Brillouin (CEA-CNRS), Ce-Saclay, 91191 Gif-sur-Yvette Cedex, France \\ PACS.61.30.Vx: Polymer liquid crystals \\ PACS.62.20.Dc: Elasticity, elastic constants \\ PACS.61.30.Hn; 68.08.Bc: Surface phenomena: anchoring, wetting \\ PACS.64.70.Md: Transition in liquid crystals
}

\begin{abstract}
Recent studies have revealed that high molecular weight liquid crystals typically exhibit shear induced phases within the isotropic melt. From rheo-birefringence measurements, we demonstrate that those shear induced phases are not coupled with conventional orientational-order fluctuations. From rheoSANS experiments, we show that the polymer chain is deformed at time scales longer than the viscoelastic relaxation time. Finally, careful visco-elastic measurements reveal a strong elastic behaviour; the melt is not a viscoelastic liquid but solid-like at equilibrium one hundred degrees over the glass transition temperature and up to $15^{\circ} \mathrm{C}$ over the Isotropic-Nematic phase transition. This supra molecular cohesion and its extra long relaxation times may explain the emergence of non-linear phenomena.
\end{abstract}

Introduction. - Flow induced phase transitions are some of the most spectacular but also of the least understood non-equilibrium phenomena. Their understanding is of practical importance for tuning the processing conditions for plastics, petroleum products, and other related materials.

Side-Chain Liquid Crystal Polymers (LC-polymers) have undergone extensive rheological investigation [1] since they were synthesised. Nevertheless, the identification of shear-induced transitions in these melts is only very recent [2]. At temperatures above the equilibrium Isotropic-Nematic transition temperature $\mathrm{T}_{\mathrm{NI}}$, and above a critical shear rate $\dot{\gamma}^{*}$, the isotropic phase transforms to a non-equilibrium nematic phase. This non-equilibrium phase is singular and is not describable as a simple shift of the Isotropic-Nematic transition temperature. Because it is an unexpected phenomenon and because of their well-characterised physico-chemistry properties, the analysis of the shear induced transition will lead us to a completely new and fundamental observation: LC-polymer melts are not viscoelastic fluids but solidlike at equilibrium. Non-linear phenomena are the consequence of this macroscopic scale cohesion. 
The paper is constructed as followed: we will determine and compare the characteristic times of a LC-polymer to the critical times associated with the shear induced phase. It will be demonstrated that the chain is deformed in the shear induced phase and that the phase is induced at time scales longer than the time scale of orientational-order fluctuations and longer than the viscoelastic relaxation time. The viscoelastic relaxation time is defined as the longest characteristic time of the melt, i.e. the time afterwards the polymer is supposed to flow and is therefore determined in the flow regime. Exploring long time scales, we will identify a strong low frequency elastic behaviour which persists from temperatures far above the Isotropic-Nematic transition (at least at $\mathrm{T}-\mathrm{T}_{\mathrm{NI}}=\Delta T=+15^{\circ} \mathrm{C}$ ) and the glass transition temperature $\left(\mathrm{T}-\mathrm{T}_{\mathrm{g}}=+100^{\circ} \mathrm{C}\right)$, down to the nematic state $\left(\Delta T=-14^{\circ} \mathrm{C}\right)$. The identification of an elastic behaviour is a fundamental result which implies that the flow regime is not the terminal behaviour and that the viscoelastic relaxation time should no more be defined as the longest relaxation time in a LCpolymer melt.

The polymer studied is a low polydisperse liquid crystalline polyacrylate (PACN) with the chemical formula:

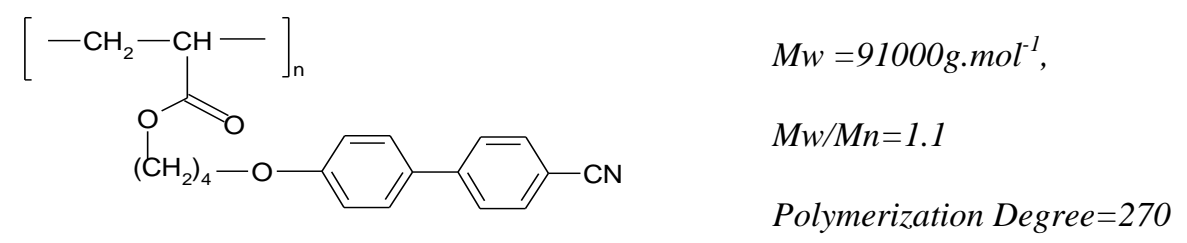

It displays the following mesophase sequence: I(isotropic) $-119^{\circ} \mathrm{C}-\mathrm{N}($ nematic $)-30^{\circ} \mathrm{C}$ - Glassy state The Isotropic-Nematic transition temperature $\mathrm{T}_{\mathrm{NI}}$ is determined by birefringence observation by increasing the temperature.

Experimental Setups. - The rheo-optical experiments were performed in transmission mode under crosspolarized microscopy (Olympus BX60) in the (velocity, neutral axis) plane. The magnification was 100X and the cross-polarizers were oriented at $45^{\circ}$ to the flow direction. Steady-state shear flow conditions were ensured using a CSS450 Linkam shear cell, home-improved to optimise the thermal environment (temperature gradient $< \pm 0.05^{\circ} \mathrm{C}$ ). Cone-plate and plate-plate geometries were tested. For the cone-plate geometry, the gap in the observation window is $80 \mu \mathrm{m}$. In the plate-plate shear cell, the gap ranges from $75 \mu \mathrm{m}$ to $125 \mu \mathrm{m}$. The birefringence was measured using a wavelength $\lambda=4700 \AA \pm 150 \AA$. The transmitted intensity $I$ was measured with a photosensitive diode and normalised to the uncrossed polarizer intensity $I_{0}$. 
Visco-elastic measurements were carried out with an ARES rheometer equipped with an air-pulsed oven and used in dynamic frequency sweep mode. This thermal environment ensures temperature control within $0.1{ }^{\circ} \mathrm{C}$. The samples were placed between plate-plate (diameter $12 \mathrm{~mm}$ ) or cone-plate (diameter $20 \mathrm{~mm}$ and angle $\left.2.25^{\circ}\right)$ fixtures. The samples were heated far above the glass transition $\left(80\right.$ to $\left.100^{\circ} \mathrm{C}\right)$, submitted to vacuum, sheared to get rid of micro-bubbles and long time thermalised before starting measurements. The molecular weight was controlled before and after the rheological investigation.

Rheo-Small Angle Neutron Scattering (Rheo-SANS) was performed on the PAXY spectrometer of the Laboratoire Léon Brillouin using a homemade Couette [3] shear-cell ((velocity-vorticity) plane). A wavelength of $8 \AA$ and a sample-multidetector distance of $2 \mathrm{~m}$ were chosen.

Results and discussion. - To identify the shear induced transition and the critical shear rate associated, the evolution of the birefringence is measured as a function of the shear rate at various temperatures and gap thicknesses. The birefringence $\left\langle\Delta n_{x}\right\rangle$ is measured in the plane (velocity, vorticity). It corresponds thus to a value averaged over the whole sample thickness (weighted of the fractions of isotropic and shear induced phases). $\left\langle\Delta n_{x}\right\rangle$ is extracted using the relationship: $I / I_{0}=\sin ^{2}\left(<\Delta n_{x}>. e . \pi / \lambda\right)$ where $e$ is the gap. At zero shear rate, a slight deviation from non-zero birefringence is observed that we attribute to the scattering from impurities. Fig. 1a shows the evolution of the birefringence from $\Delta T=+1{ }^{\circ} \mathrm{C}$ up to $\Delta T=+9^{\circ} \mathrm{C}$ above $\mathrm{T}_{\mathrm{NI}}$ and for a gap thickness $e=100 \mu \mathrm{m}$ in steady shear conditions. It reveals typically [2] a strong non-linear behaviour with, above a critical shear rate $\dot{\gamma}^{*}$, an abrupt increase of the birefringence.

Two regimes can be distinguished. The low-shear rate regime depends weakly on the shear rate. It is similar to the conventional flow effect on the orientational-order fluctuations, called paranematic phase [4] or flow birefringence [5]. The second regime is defined by an abrupt departure of the birefringence above a critical shear rate (non-linear regime). This regime cannot be explained conventionally. It corresponds to a strong discontinuity in the rheo-optical behaviour and reveals the appearance of a nonequilibrium first-order phase transition. As already observed on other LC-polymers [2], this non-linear behaviour is characterised by an alignment of the director along the velocity axis and a texture free from defects. It indicates the shear induced appearance, for $\dot{\gamma}>\dot{\gamma}^{*}$, of a non-equilibrium nematic phase within the isotropic phase. Fig.1b gives an estimation of the critical shear rate $\dot{\gamma}^{*}$, determined by the intersection of the baseline and the tangent to the curve at the departure of the birefringence. Its inverse defines a (non- 
equilibrium) characteristic time $\tau^{*}=1 / \dot{\gamma}^{*}$. In the present case, $\tau^{*}$ is of the order of tenths of second. This result is independent of the thickness of the sheared sample and of the geometry (cone-plate and plateplate provide similar results). Fig.1c displays the birefringence versus shear rate for different gaps ranging from $75 \mu \mathrm{m}$ to $125 \mu \mathrm{m}$ at $\Delta T=+1^{\circ} \mathrm{C}$. This time scale is very large with respect to pretransitional dynamics [6]. Indeed, recent measurements [7] on a LC-polyacrylate presenting the same liquid crystal moiety (cyanobiphenyl) but a longer spacer and, a similar molecular weight indicate similarly critical shear rates of about $\dot{\gamma}^{*}=10 \mathrm{~s}^{-1}$ at $\Delta T=+1^{\circ} \mathrm{C}$, whereas the orientational-order fluctuation life time, $\tau_{\text {fluct }}$, is about $\tau_{\text {fluct }}\left(\Delta \mathrm{T}=+1^{\circ} \mathrm{C}\right)=10^{-4} \mathrm{~s}[6]$. The discrepancy between $\tau^{*}$ and $\tau_{\text {fluct }}$ is very important; $\tau^{*}$ is about $10^{3}$ times larger than $\tau_{\text {fluct }}$. It excludes a coupling with these orientational-order fluctuations.

We now examine the rheological spectrum. Completely new rheological features can be extracted by examining the low frequency behaviour. This leads to our main result; the LC-polymer is not a viscoelastic fluid.

Let's describe the measurements obtained from high down to low temperatures. Fig.2a to c display the frequency dependence of the storage modulus $G^{\prime}(\omega)$ from $\Delta T=+15^{\circ} \mathrm{C}$ down to $\Delta T=-8^{\circ} \mathrm{C}$ with respect to the Isotropic-Nematic transition temperature. $G^{\prime \prime}(\omega)$ is not systematically represented since it displays the conventional $\omega$ scaling dependence whatever the strain (inset of Fig.2b). The evolution of the elastic modulus reveals a completely new and non trivial behaviour. Instead of a low frequency flow behaviour, the modulus displays a huge elastic plateau; the polymer is able to exhibit a solid-like behaviour within the isotropic phase. Two regimes can be distinguished. At low strain amplitudes, the elastic plateau is relatively independent of the strain; it defines the regime of linear responses. Whereas at high strain amplitudes, the modulus of the elastic plateau becomes strongly strain dependent defining the non-linear regime.

Fig.3 illustrates the evolution of the elastic plateau $G_{p}$ ' (arithmetic value averaged on the frequency interval where the modulus is constant) as a function of the strain amplitude in the isotropic phase at $\Delta T=+6^{\circ} \mathrm{C}$. The linear and the non-linear regimes of the elastic response are noted " $O_{L}$ " and " $O_{N L}$ " respectively, $G_{N L}$ ' being the non-linear elastic modulus. The regime " $O$ "precedes in terms of strain and frequency the classical linear viscoelastic regime noted "regime $I$ " $\left(I_{L}\right)$. It corresponds to a so far unreported solid-like response using a conventional rheometer. Since the regions " $O_{L}$ " and " $O_{N L}$ " 
correspond to plateau moduli, they are not frequency dependent whereas the entrance in the regime " $I_{L}$ " is frequency dependent (conventional linear viscoelastic behaviour). These results contrast with the conventional flow behaviour [1]. They are indifferently obtained by scanning from high to low frequency and are qualitatively independent of the geometry (plate-plate and cone-plate geometries were tested). In order to display the elastic behaviour, a non-standard protocol has been elaborated which consists essentially in optimising the polymer/substrate interactions to avoid any slippage. Indeed, the elastic plateau is emerging from the background noise by applying long equilibration time (days) and thermal processes, by testing different substrates and once the micro bubbles within the polymer melt are removed. These conditions are not fulfilled in standard measurements.

To our knowledge, the only work reporting on drastic polymer/substrate anchoring conditions is a piezorheometer study, carried out by Martinoty and coworkers who identified also an unexpected elastic response up to $50 \mu \mathrm{m}$ in the isotropic phase in LC-Polymers [8]. Our present results corroborate and extend at larger scale $(200 \mu \mathrm{m})$ and using a conventional rheometer, this pioneering study. These piezorheometer measurements have shown that the viscoelastic behaviour evolves continuously from a classical flow behaviour to a solid-like behaviour by decreasing the thickness down to $50 \mu \mathrm{m}$ for LCpolymers. It was interpreted by the existence of size-dispersed elastic clusters reminiscent of the glass transition. The previous authors report on either an elastic behaviour obtained at low thickness using glass scratched substrates or a conventional viscous behaviour obtained when the polymer/substrate interactions are weak. They do not report on non-linear responses. We observe in contrast that, over the critical strain, the system exhibits a non-linear elastic behaviour. We interpret this regime as the entrance into an intermediate slippage regime combining both elastic and partial modulus lost responses. In other words, the dissipative sliding mode is superposed to the initial linear elastic response, giving rise to a non-linear response. From this point of view, both linear and non-linear responses probe the elastic character of the material; the sliding ability being also a characteristic feature of a solid-like property.

Thus, anchoring and small strain amplitudes are the necessarily conditions to obtain the linear elastic response. In the case of the LC-polymers, we suspect that the anchoring property is particularly strong since the contact to the solid surface is created via an interfacial liquid crystal layer [5,9]. This leads us to our fundamental result: in a chemically homogeneous melt, the viscoelastic relaxation time is not the 
longest time. The strong elastic character implies a macroscopic cohesion and corresponding extra long relaxation times.

Let's express the constitutive equations for the elastic modulus $G$ ' in the linear (1) and the non-linear regime (2):

-at $\gamma<\gamma_{N L}$ : linear regime: $G^{\prime}=G_{0}{ }^{\prime}+\eta \cdot \tau \cdot \omega^{2} /\left(1+\omega^{2} \cdot \tau^{2}\right)$.

-at $\gamma>\gamma_{N L}:$ non-linear regime: $G^{\prime}=G_{N L}{ }^{\prime}+\eta \cdot \tau \cdot \omega^{2} /\left(1+\omega^{2} \cdot \tau^{2}\right)$,

where $G_{N L}{ }^{\prime}=G_{0}{ }^{\prime} \cdot \exp \left[-\left(\gamma-\gamma_{N L} / \gamma_{N L}\right)\right]^{\alpha}$; and $G_{0}{ }^{\prime}$ and $\gamma_{N L}$ are the non-perturbed (linear) modulus and strain of the solid-like response. $\alpha$ is a dissipation exponent illustrating the decrease in modulus due to the sliding of the solid-like response. $\tau$, the viscoelastic relaxation time is obtained by the interception at high frequencies of $G^{\prime}$ and $G^{\prime \prime}$ fitting with $\omega^{2}$ and $\omega$ scaling respectively (flow regime) in the linear viscoelastic regime. In Fig. $3, G_{0}{ }^{\prime}$ and $\gamma_{N L}$ are about $10^{3} \mathrm{~Pa}$ and $2 \%$ respectively.

Let's now focus on the influence of the temperature on the evolution of the solid-like behaviour. Fig. 4 displays the variation of $G_{0}{ }^{\prime}(T)$ as a function of temperature in the linear regime at $\gamma=1 \%$. The elastic plateau is weakly temperature dependent within our observation temperature window $\left(25^{\circ} \mathrm{C}\right)$. One notices a slight softening tendency at high temperature. Temperatures over $\Delta T=+15^{\circ} \mathrm{C}$ were not tempted because of a possible thermal degradation of the polyacrylate ester links [10].

The huge value of the elastic plateau, its persistence through and far above the Isotropic-Nematic transition (at least up to $15^{\circ} \mathrm{C}$ ) show that it can be interpreted neither as a phase pretransitional dynamics nor as a mesomorphic property. This reveals a strong and macroscopic cohesion within the isotropic melt never been revealed at such a scale (the gap thickness in the plate-plate geometry is $200 \mu \mathrm{m}$ ). In contrast with plateaus originating from the formation of a physical network or heterogeneities within a polymer melt, a gelation, an ordering transition (associative polymers) [11], the elastic response of LC-polymers is not due to a cross-linking (the molecular weight keeps constant before and after the rheological investigations) or a chemical heterogeneity.

At high strain amplitudes and/or low temperature (conditions of high dynamic friction), $G_{N L}{ }^{\prime}(\omega)$ and $G$ ” $(\omega)$ moduli become progressively independent of the strain and the conventional viscoelastic behaviour of polymers is reached. Inset of Fig.2b displays the frequency dependence of $G^{\prime}(\omega)$ and $G^{\prime \prime}(\omega)$ at $\Delta T=+6^{\circ} \mathrm{C}$. The extrapolated viscoelastic relaxation time $\tau\left(\Delta T=+6^{\circ} \mathrm{C}\right)$ is about $2.5 .10^{-3} \mathrm{~s}$. This indicates that 
the chain would be distorted for shear rates beyond $400 \mathrm{~s}^{-1}$. The comparison with the critical shear rate of the induced phase $\left(\dot{\gamma}^{*}\left(\Delta T=+6^{\circ} \mathrm{C}\right) \approx 15 \mathrm{~s}^{-1}\right)$ shows that the phase is induced at times scales longer that the viscoelastic relaxation time. This time scale incompatibility is consistent with a previous study on another LC-polymer but possessing a broad polydispersity [7]. Here, the low polydispersity excludes any shear induced effect related to a selective molecular weight stretching [12].

Finally, we report rheo-SANS experiments carried out on this melt (made of hydrogenated and main-chain deuterated LC-polymers [13]), to determine the non-equilibrium main-chain conformation. The LC-polymer main-chain evolution at $\Delta T=+1^{\circ} \mathrm{C}$ under shear flow is displayed on Fig.4 [14]. From $\dot{\gamma} \geq 10 \mathrm{~s}^{-1}$, the chain is deformed and keeps a constant anisotropy of about: $R_{v} / R_{z} \geq 12 \%$, where $R_{v}$ and $R_{z}$ are the components of the radius of gyration along and perpendicularly (neutral axis) to the velocity respectively. The anisotropy at shear rate values significantly much lower ( 2 orders of magnitude) than the terminal time zone, confirms that larger time scales and correlatively larger structures than a single chain are involved.

This structural observation, the shear induced phase transition and the elastic plateau are the evidence of supramolecular scales within the isotropic LC-Polymer. Because of an excellent anchoring probably enhanced by the strong liquid crystal/substrate interactions, the elastic response was visible in the present experiments. Since this behaviour is insensitive to the Isotropic-Nematic transition and since it can be revealed even far from it, it is not a mesomorphic but a melt property which should be observable in other viscoelastic materials [7]. Finally, it should be noted that LC-polymers present architectures similar to branched polymers. An upturn in $G^{\prime}$ is theoretically predicted in branched polymers [15]. Nevertheless, the liquid-crystal side-chains do not bring by themselves an elastic response (low molecular weight liquid crystals are viscous). But the friction factor and the polar interaction effects play certainly a role in the giant elasticity observed here.

Conclusions.-In this paper, we first focused on the origin of shear-induced phase in LC-polymer melts. This spectacular non-linear phenomenon has allowed us to establish new fundamental features. First, the shear-induced phase cannot be due to a coupling to the orientational-order parameter fluctuations. Second, even far above the Isotropic-Nematic transition, and far above the glass transition, LC-polymers present a huge macroscopic cohesion $\left(G_{0}{ }^{\prime}>10^{3} \mathrm{~Pa}\right)$. Third, rheo-SANS experiments conducted in the isotropic melt 
have demonstrated that the chain is deformed at times scales longer than the viscoelastic the terminal time. These observations imply that extra long length and time scales exist in the melt which do not originate from mesomorphic properties. Finally, our present study supports and extends at a much larger macroscopic scale, the first observations of elasticity carried out with a piezorheometer [8]. Indeed, from the comparison of the results obtained with two different techniques, using different substrates, with different polymers, at different temperatures away from transition temperatures, similar solid-state properties have been evidenced. These similarities demonstrate the fundamental elastic character of LCpolymers which are wrongly considered as flowing fluids above the glass transition temperature. It is also interesting to note that previous studies [16], regarding the low stress creep behaviour of a nematic mainchain liquid crystalline polymer, had also reported on a solid-like behaviour at $\mathrm{T}<\mathrm{T}_{\mathrm{NI}}$. It was interpreted as related to smectic or nematic texture defects. These defects play certainly a role in the solid-like response in the nematic and smectic phases but our results invalidate the interpretation in terms of defects since the elasticity is also identified in the isotropic phase (up to $+15^{\circ} \mathrm{C}$ above $\mathrm{T}_{\mathrm{NI}}$ ). At this temperature, the defects associated to the monodomains have disappeared through the nematic to isotropic phase transition (see inset of Fig.1c). Any phase or phase defect effect is excluded to explain such a huge elasticity within the isotropic phase of a non-entangled liquid crystalline polymer. One fundamental consequence of this present experimental observation is that, far above phase and glass transitions, longer times than the socalled viscoelastic terminal time exist and have to be taken into consideration to explain the LC-polymer behaviour, in particular non-linear phenomena such as the shear induced phase. 


\section{REFERENCES}

[1] RuBIN S.F. et al., Macromol., 28 (1995) 3521; CoLbY R.H. et al Liquid Crystals, 13 (1993) 233;

ZENTEl R. et al., Makromol. Chem, 187 (1986) 1727; WeWERKA A. et al., Rheol.Acta, 40 (2001) 416; LEE K.M. et al., Macromol,.36 (2003) 8796.

[2] Pujolle-Robic C., Noirez L., Nature, 409 (2001) 167; Pujolle-Robic C., Olsmted P.D., NOIREZ L., Europhys. Lett., 59 (2002) 364.

[3] BARONi P., PUJOLle-Robic C. \& NoIREZ L., Rev.Sci.Instrum., 72 (2001) 1.

[4] Hess S., NAturforsch Z., 31a (1976) 1507; OnUki A, Kawasaki K., Ann. Physics, 121 (1979) 456

OlMSted P.D., GoldBART P., Phys. Rev. A41, (1990) 4578; ibid A46 (1992) 4966.

[5] de Gennes P.G. \& Prost J., The Physics of Liquid Crystals (Oxford Science Publications, 1993).

[6] ReYs V. et al., Phys. Rev. Lett., 61 (1988) 2340.

[7] NoIREZ L. submitted to Phys. Rev. E.

[8] Gallani J.L. et al., Phys. Rev. Lett, 72 (1994) 2109; Martinoty P. et al. Macromol. 32 (1999) 1746 ; Collin D. et al., Physica A 320 (2002) 235.

[9] Jerome B., J. Phys.: Condens. Matter, 6 (1994) A269 ; Jerome B., Commandeur J., de Jeu W.H., Liq. Cryst., 22 (1997) 685.

[10] Grassie N. et al. J. Polym. Sci., A-1, 9 (1971) 931.

[11] AOKI Y., Macromol., 20 (1987) 2208 ; NEUGEBAUER D. et al. Macromol., 36 (2003) 6746; TAE G. et al, Macromol., 35 (2002) 4448, WATAnABE H. et al, Macromol., 34 (2001) 6742; Bousmina M., Rheol.Acta, 38 (1999) 251.

[12] Brochard-Wyart F. \& de GenNes P. G., C.R. Acad. Sci. Paris, 306 (1988) 699.

[13] Higgins J.S. \& Benoît H.C., Polymers \& Neutron Scattering, (Oxford Science Publications, 1994).

[14] PUjolLe-Robic C., Ph-D Thesis, University ParisXI, 2002.

[15] MCLeISH T.C.B., MiLner S.T., Advances in Polymer Science, 143 (1999).

[16] COLBY R.H. et al., Europhys. Lett., 54 (2001) 269 
Figure captions:

Fig.1a-Evolution of the birefringence $\left\langle\Delta n_{x}\right\rangle$ as a function of the shear rate $(\dot{\gamma})$ at: $(\bullet): \Delta T=+1^{\circ} \mathrm{C},(\Delta)$ : $+3^{\circ} \mathrm{C},(\square):+5^{\circ} \mathrm{C},(\square):+7^{\circ} \mathrm{C},(+):+9^{\circ} \mathrm{C}$ above $\mathrm{T}_{\mathrm{Nl}}=119^{\circ} \mathrm{C}$ for $100 \mu \mathrm{m}$ gap thickness (plate-plate shear geometry)

Fig. $1 \mathrm{~b}$-Critical shear rate $\dot{\gamma}^{*}$ as a function of the temperature at $\Delta T=+1^{\circ} \mathrm{C},+3^{\circ} \mathrm{C},+5^{\circ} \mathrm{C},+7^{\circ} \mathrm{C},+9^{\circ} \mathrm{C}$.

Fig.1c- Influence of the gap thickness $((O) 75 \mu \mathrm{m} ;(\square) 100 \mu \mathrm{m} ;(\triangle) 125 \mu \mathrm{m})$ on the shear induced birefringence at $\Delta T=+1{ }^{\circ} \mathrm{C}$ above $\mathrm{T}_{\mathrm{NI}}$. The black inset represents the isotropic phase at rest; the blue inset represents the shear induced nematic phase. The director is aligned along the velocity direction.

Fig.2a-c: Frequency dependence of the viscoelastic elastic modulus $G^{\prime}(\omega)$ above and below $\mathrm{T}_{\mathrm{NI}}$ (plateplate geometry) at different strain amplitude. The viscous modulus $G$ " $(\omega)$, fitting with $\omega$ scaling, is represented in the inset only.-2a: $\Delta T=+15^{\circ} \mathrm{C}, 2 \mathrm{~b}: \Delta T=+6^{\circ} \mathrm{C}$. The inset displays $G^{\prime \prime}(\omega)$ and $G^{\prime}(\omega)$ fitting with $\omega$ and $\omega^{2}$ scaling respectively (cone-plate fixture-20mm diameter, $2.25^{\circ}$ ). The straight lines are guides for the eyes, $2 \mathrm{c}: \Delta T=-8^{\circ} \mathrm{C}$.

Fig.3. Evolution of the elastic plateau modulus as a function of the strain at $\Delta T=+6^{\circ} \mathrm{C}$ (regime " $I_{L}$ " is measured at $0.1 \mathrm{rd} / \mathrm{s})$.

Fig.4. Evolution of $G_{0}$ ' versus temperature in the linear regime of the elastic response at $\gamma=1 \%$.

Fig.5. Evolution as a function of the shear rate at $\Delta T=+1^{\circ} \mathrm{C}$ of the components following the velocity axis $(\circ)$ and the neutral axis $(\bullet)$ of the radius of gyration of the polymer main-chain. The dotted lines are guides for the eyes. 

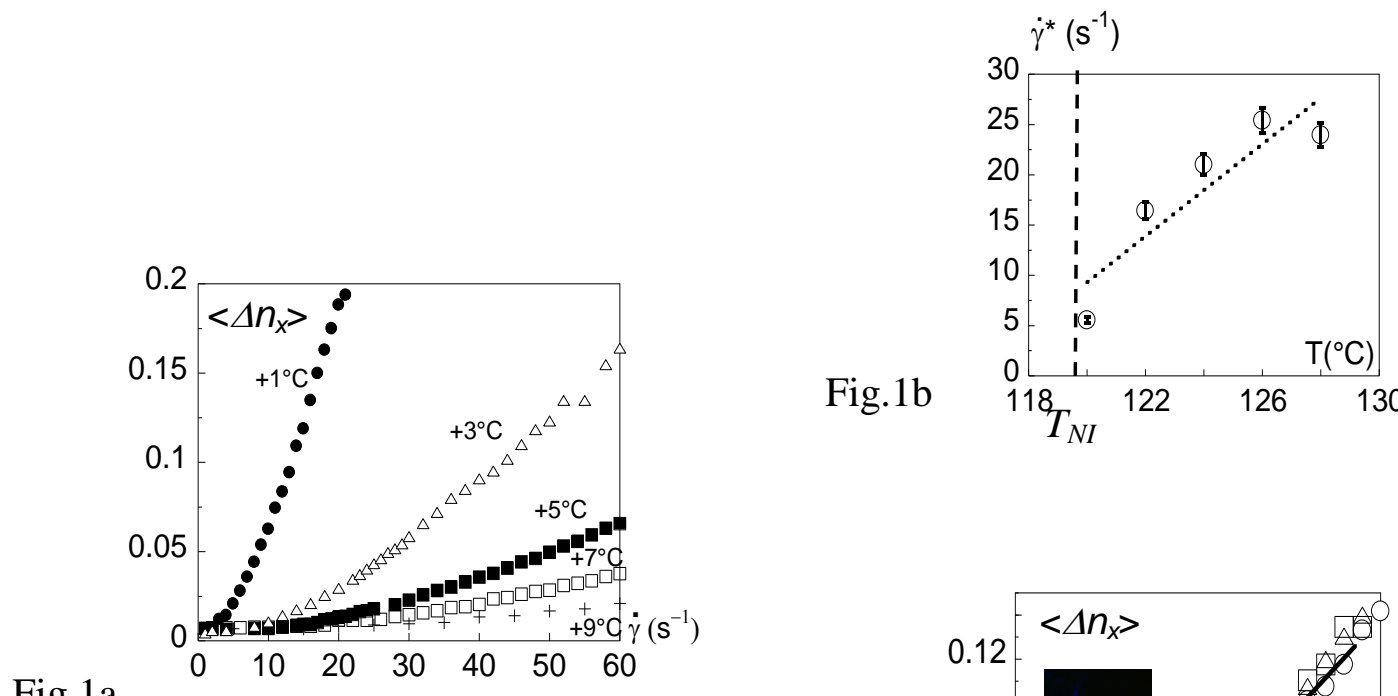

Fig.1a

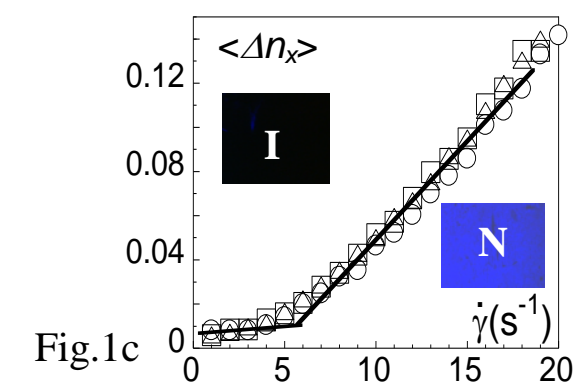



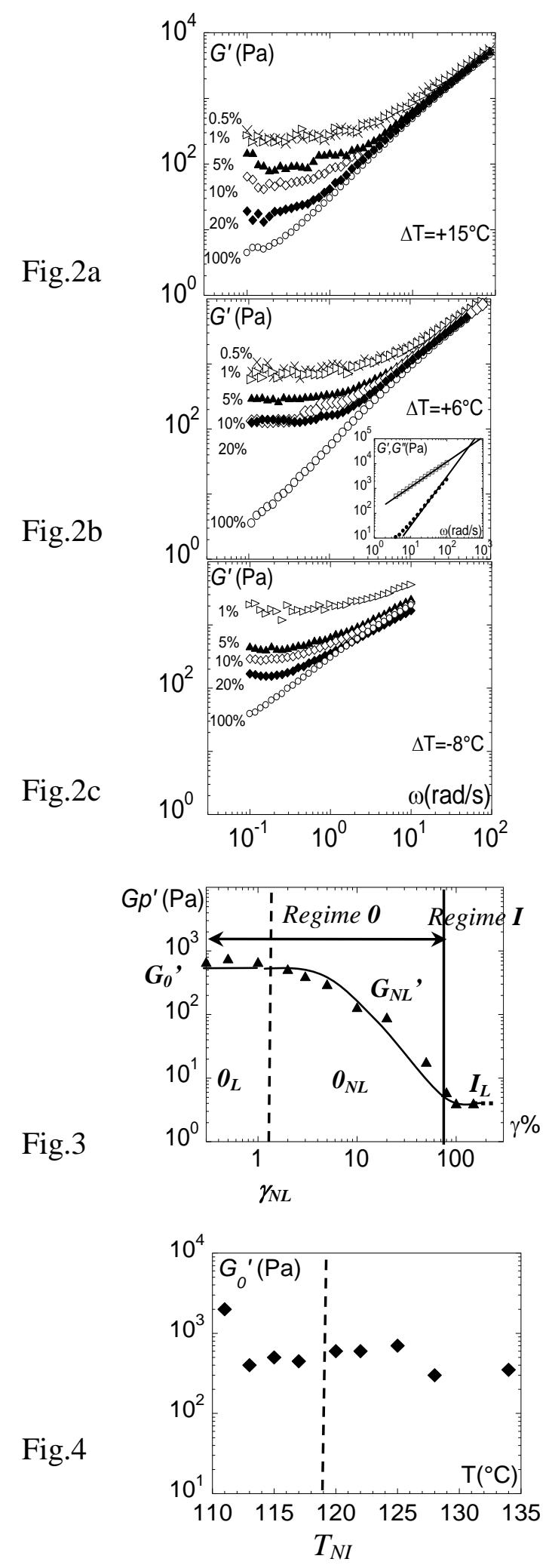


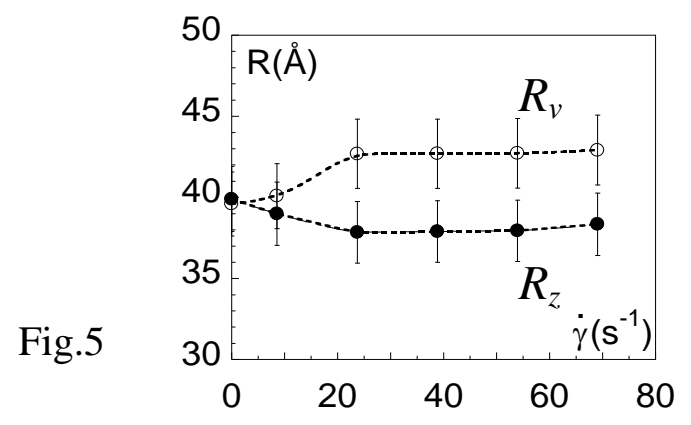

